\section{Comparison of post-remission strategies in acute myeloid leukemia: Autologous hematopoietic stem cell transplantation versus consolidation chemotherapy}

Zeynep Arzu Yegin, Asena Dikyar, Lale Aydın Kaynar, Ferda Can, Zübeyde Nur Özkurt, Münci Yağcı

Department of Hematology, Gazi University School of Medicine, Ankara, Turkey

\begin{abstract}
Autologous Hematopoietic Stem Cell Transplantation (auto-HSCT) has become a therapeutic option for first-line consolidation in Acute Myeloid Leukemia (AML) patients with favorable and intermediate risk features. A total of 101 AML patients in first complete remission, who were not eligible for allogeneic HSCT, were randomized to receive intensive cytarabine-based chemotherapy or to undergo auto-HSCT. The probability of LFS was significantly better in auto-HSCT recipients compared to chemotherapy arm $(43 \%$ vs $4.8 \%, \mathrm{p}=0.008)$. At the end of 915 (30-4470) days of followup, the probability of overall survival was better in auto-HSCT group compared to chemotherapy, without statistical significance $(79.2 \%$ vs $38.8 \%, \mathrm{p}=0.054)$. Multivariate analysis revealed a significant predictive impact of cytogenetic risk status on OS ( $\mathrm{p}=0.002$, HR: $2.824,95 \%$ CI: 1.445 5.521). Auto-HSCT is considered as an effective consolidation approach in favorable and intermadiate risk AML patients.
\end{abstract}

\section{Introduction}

Maintenance of remission is considered as a major concern in the primary treatment of Acute Myeloid Leukemia (AML) despite favorable remission rates after induction treatment. Complete Remission (CR) rates are approximately $70-80 \%$ for adult patients with AML, however $60 \%$ of these patients experience relapse in their first $\mathrm{CR}$ on intensive chemotherapy with cytarabine based regimens. ${ }^{1}$ As a result of progressive efforts to improve Leukemia Free Survival (LFS) in AML patients, distinct therapeutic approaches for post-remission consolidation have been developed. Treatment decision is mainly based on cytogenetic risk profile, quality and intensity of remission including Minimal Residual Disease (MRD) determination and patient related factors to optimize potential options for salvage therapy. $^{2}$

Currently available consolidation strategies for AML patients in CR1 are intensive chemotherapy, autologous (auto) and allogeneic (allo) Hematopoietic Stem Cell Transplantations (HSCT). Although allo-HSCT is the most effective treatment to prevent leukemia relapse, high Non Relapse Mortality (NRM) rate, which is mainly associated with Graft versus Host Disease (GvHD), remains to be the major obstacle in allo-HSCT recipients. In this perspective, auto-HSCT has become a therapeutic option for first-line consolidation in AML patients with favorable and intermediate risk features as it prolongs LFS with a tolerable toxicity profile compared to allo-HSCT. ${ }^{3-5}$ Nevertheless, lack of Graft versus Leukemia (GvL) effect and possibility of graft contamination may increase post-transplant relapse incidence. Minimal residual disease eradication at the time of transplant may help to improve prognosis in this group of patients. ${ }^{3,6-8}$

This retrospective study was planned to compare the efficacy of auto-HSCT with respect to conventional cytarabine based chemotherapy in a relatively elderly population of AML patients in CR1.

\section{Materials and Methods}

A total of 101 AML patients in CR1 [median age: 47(19-79); male/female: 51/50], who were not eligible for allo-HSCT and did not have an HLA compatible donor, were randomized to receive intensive cytarabine-based chemotherapy or to undergo auto-HSCT after high dose cyclophosphamide and busulfan as conditioning regimen. Only one patient received TEAM (thiotepa, etoposide, cytarabine, melphalan) conditioning regimen due to neurotoxicity secondary to central nervous system leukemia. Diagnosis and risk stratification were based on FrenchAmerican-British classification and European Leukemia Net 2017 guidelines. ${ }^{9}$ Patient characteristics are summarized in Table 1.

Continuous variables were compared using T-test, Mann Whitney U and Kruskal Wallis tests while Chi-square test was used for categorical variables. Correlation analysis was performed using Pearson and Spearman tests. Kaplan Meier and log rank tests were used for survival analysis. Risk factors for survival were determined by Cox
Correspondence: Zeynep Arzu Yegin, Gazi University School of Medicine, Department of Hematology, Beşevler, Ankara, Turkey. Tel.: +90.3122025579 - Fax: +90.3122236714

E-mail: zeyneparzuyegin@gmail.com

Key words: Acute myeloid leukemia; autologous hematopoietic stem cell transplantation; consolidation chemotherapy; leukemia relapse; prognosis.

Contributions: ZAY: Hypothesis and design, data analysis, interpretation of findings, manuscript writing; $\mathrm{AD}$ : Acquisition of data, interpretation of findings; LAK: Acquisition of data; FC: Acquisition of data; ZNO: Hypothesis, manuscript review; MY: Hypothesis, manuscript review.

Conflict of interest: The authors declare no conflict of interest.

Ethics approval: The study was approved by the local ethical commitee of Gazi Medical School

Consent for publication: Consent has been obtained.

Availability of data and materials: Available by authors.

Funding: None.

Conference Presentation: $7^{\text {th }}$ International Congress on Leukemia, Lymphoma, Myeloma, İstanbul, Turkey, May 2019. Oral presentation, No: OP-012.

Received for publication: 15 November 2019 Accepted for publication: 10 July 2020 .

This work is licensed under a Creative Commons Attribution-NonCommercial 4.0 International License (CC BY-NC 4.0).

(C) Copyright: the Author(s), 2020

Licensee PAGEPress, Italy

Hematology Reports 2020; 12:8380

doi:10.4081/hr.2020.8380

regression test. SPSS 22.0 (SPSS Inc, Chicago, IL, USA) programme was used for statistical analysis and $\mathrm{p}<0.05$ was considered as statistically significant. The study was approved by the local ethical commitee of Gazi Medical School.

\section{Results and Discussion}

Leukemia relapse was demonstrated in 22 patients $(31.4 \%)$ in the chemotherapy arm at median 330 (60-2190) days of follow-up. In this group, allo-HSCT was performed in 
4 patients $(18.2 \%)$ as salvage treatment for post auto-HSCT relapse. Among auto-HSCT recipients, leukemia relapse was observed in 14 patients (45.2\%) at median 225 (60-395) days of follow-up. Allo-HSCT was performed in a total of 12 patients $(85.7 \%)$ who had experienced relapse after autoHSCT. Five-year relapse incidence was found to be $65 \%$ and $46 \%$, in chemotherapy and auto-HSCT groups respectively $(p>0.05)$. A total of 52 patients were analysed for LFS. The probability of LFS was significantly better in auto-HSCT recipients compared to chemotherapy arm (43\% vs 4.8\%, $\mathrm{p}=0.008)$. One-year Non Relapse Mortality (NRM) rate was $27 \%$ in chemotherapy and $22 \%$ in auto-HSCT groups ( $p>0.05)$. At the end of 915(30-4470) days of follow-up, the probability of Overall Survival (OS) was better in auto-HSCT group compared to chemotherapy, without statistical significance $(79.2 \%$ vs $38.8 \%$, $\mathrm{p}=0.054$ ) (Figure 1). Univariate and multivariate analysis revealed a significant predictive impact of cytogenetic risk status on OS ( $\mathrm{p}=0.002$, HR: $2.824,95 \%$ CI: $1.445-$ 5.521). This retrospective study yielded comparable results with the previous reports, which evaluate consolidation strategies in AML patients. Although leukemia relapse was seen more frequent in auto-HSCT recipients in short-term follow-up, 5-year relapse probability was found to be higher in chemotherapy arm without statistical significance. In addition, LFS was significantly longer in auto-HSCT patients with similar NRM rates in both groups. However, better OS in auto-HSCT arm did not reach statistical significance, which may be due to small sample size and short followup. Several studies have investigated the role of auto-HSCT in AML consolidation therapy. In a study by Vellenga et al., autoHSCT group represented a reduced relapse rate and better relapse-free survival compared to conventional chemotherapy. However, NRM was found to be higher in auto-HSCT group. Statistical insignificance in terms of OS was primarily based on different post-transplant salvage strategies including allo-HSCT. ${ }^{10}$ In a retrospective analysis of European Society for Blood and Marrow Transplantation (EBMT) in 3567 AML patients who underwent auto-HSCT, the probability of relapse at 10 years was $16 \%$ which was markedly lower compared to our study. Similarly, a lower NRM rate (8\%) and a better LFS (76\%) were reported in the same study. Use of mobilized blood and older age were found to be associated with increased risk of relapse and decreased probability of LFS. $^{3}$ Leukemia relapse, which affects approximately $40-50 \%$ of auto-HSCT recipients with AML in CR1 and

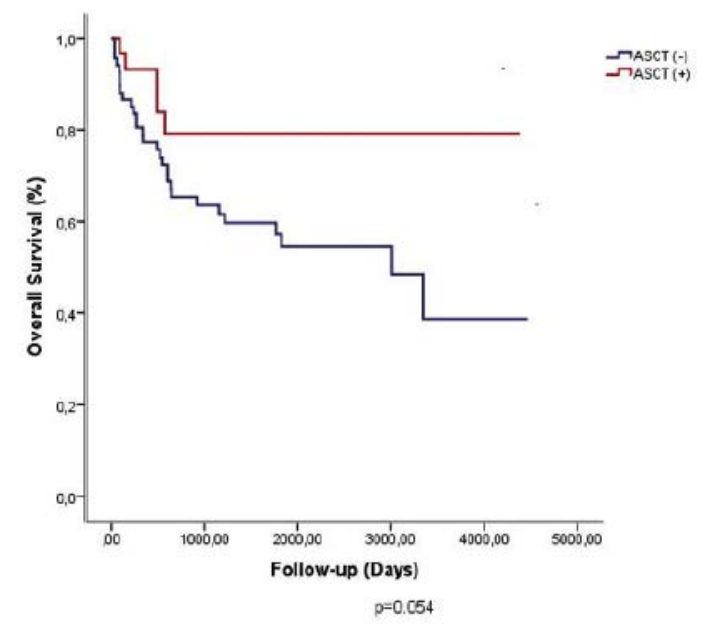

Figure 1. The probability of overall survival was better in auto-HSCT group compared to chemotherapy group, without statistical significance $(79.2 \%$ vs $38.8 \%, p=0.054)$.

Table 1. Patient characteristics.

\begin{tabular}{|c|c|}
\hline Characteristic & N. \\
\hline $\begin{array}{l}\text { All patients, } \mathrm{n}(\%) \\
\text { Consolidation chemotherapy } \\
\text { Autologous hematopoietic stem cell transplantation }\end{array}$ & $\begin{array}{l}101(100) \\
70(69.3) \\
31(30.7)\end{array}$ \\
\hline Median follow-up, median (range) days & $915(30-4470)$ \\
\hline Age, median (range) years & $47(19-79)$ \\
\hline Gender, n (male/female) & $51 / 50$ \\
\hline $\begin{array}{l}\text { AML subtype (FAB classification), n(\%) } \\
\text { M0 } \\
\text { M1 } \\
\text { M2 } \\
\text { M4 } \\
\text { M5 } \\
\text { Unclassified }\end{array}$ & $\begin{array}{c}8(7.9) \\
13(12.9) \\
18(17.8) \\
31(30.7) \\
9(8.9) \\
22(21.8)\end{array}$ \\
\hline $\begin{array}{l}\text { Cytogenetic risk group, } \mathrm{n}(\%) \\
\text { Low } \\
\text { Intermediate } \\
\text { High }\end{array}$ & $\begin{array}{l}12(11.9) \\
45(44.5) \\
44(43.6)\end{array}$ \\
\hline Extramedullary disease, $\mathrm{n}(\%)$ & $5(4.9)$ \\
\hline CNS involvement, $\mathrm{n}(\%)$ & $3(2.9)$ \\
\hline Time from diagnosis to transplant, median (range) days & $95(57-187)$ \\
\hline Pre-transplant performance status (ECOG), median (range) & $0(0-1)$ \\
\hline Pre-transplant comorbidity index (Sorror's), median (range) & $0(0-2)$ \\
\hline $\begin{array}{l}\text { Mobilization regimen, } \mathrm{n}(\%) \\
\text { HDAC } \\
\text { IDAC } \\
\text { Cy-Etoposid } \\
\text { G-CSF }\end{array}$ & $\begin{array}{l}22(71) \\
7(22.6) \\
1(3.2) \\
1(3.2)\end{array}$ \\
\hline $\begin{array}{l}\text { Conditioning regimen, } \mathrm{n}(\%) \\
\mathrm{Cy} \mathrm{Bu} \\
\text { TEAM }\end{array}$ & $\begin{array}{c}30(96.8) \\
1(3.2)\end{array}$ \\
\hline $\begin{array}{l}\text { Stem cell source, } n(\%) \\
\text { Bone marrow } \\
\text { Peripheral blood }\end{array}$ & $\begin{array}{c}0 \\
31(100)\end{array}$ \\
\hline Number of infused CD $34^{+}$cells, median (range, $10^{6} / \mathrm{kg}$ ) & $4.47(2.2-5.86)$ \\
\hline Neutrophil egraftment, median (range) days & $12(9-27)$ \\
\hline Platelet engraftment, median (range) (days) & $13(10-202)$ \\
\hline Sinusoidal obstruction syndrome, $\mathrm{n}(\%)$ & $1(3.2)$ \\
\hline Mucositis grade, median(range) & $1(1-3)$ \\
\hline
\end{tabular}


$70 \%$ in CR2, is the major cause of treatment failure after auto-HSCT in the first 2 years. Post-transplant tumor control measures such as maintenance therapy with hypomethylating agents may be considered to overcome early relapses within the first 2 years after HSCT. Our results are compatible with the previous studies which demonstrated an advantage for auto-HSCT compared to conventional chemotherapy in terms of improved LFS with no significant impact on OS. $2,3,5,7,11,12$ In our study, the follow-up period of auto-HSCT recipients was relatively shorter than chemotherapy arm, which may be an explanation for the higher early relapse rates. We did not perform MRD analysis at the time of transplant, therefore the potential impact of MRD status on relapse incidence should also be taken into account. Similarly, higher NRM rates in our study may be associated with the higher number of elderly patients in our study population, as older age may have an adverse prognostic impact despite contradictory reports which underline the safety and efficacy of auto-HSCT in AML patients above 65 years..$^{3,4,6,13}$

Allogeneic HSCT has favorable outcomes in AML-CR1 patients with intermediate or poor cytogenetic risk profile. Intermediate risk AML patients who underwent matched sibling donor (MSD) allo-HSCT in CR1, represented best outcomes in terms of LFS and OS compared to auto-HSCT and chemotherapy arms. Nevertheless, in intermediate risk patients lacking a MSD, auto-HSCT should be considered as a valid option as better survival appears to be provided by autoHSCT compared to mismatched unrelated transplants. ${ }^{1,8,14-17}$ The main disadvantages of auto-HSCT are the possibility of contamination of leukemic cells in the stem cell product and lack of GvL effect, which causes a lower curative potential compared to allo-HSCT. ${ }^{1}$

The role of allo-HSCT as salvage treatment should be considered in AML patients who experience leukemia relapse after auto-HSCT. Approximately 20\% of autografted patients have received a second allo-HSCT with a LFS of $30 \%$ at 3 years. Younger age, late relapse, and the absence of total body irradiation in the auto-HSCT conditioning regimen have been indicated as favorable prognostic factors. Disease status at the time of allo-HSCT is considered as the most significant prognostic factor. ${ }^{12,18}$ Relatively higher incidence of salvage alloHSCT may have positively affected the long term outcomes of auto-HSCT survivors in our study.

\section{Conclusions}

In conclusion, auto-HSCT is considered as an effective consolidation approach in favorable and intermadiate risk AML patients. Salvage allo-HSCT for leukemia relapse in autografted patients remains to be a feasible treatment option which prolongs LFS and reduces relapse rates. Pre-transplant MRD negativity is critical in order to minimize graft contamination with leukemic stem cells. Post-transplant maintenance strategies may have a role in preventing relapse and improving long term outcomes of auto-HSCT.

\section{References}

1. Mizutani M, Hara M, Fujita H, et al. Comparable outcomes between autologous and allogeneic transplant for adult acute myeloid leukemia in first CR. Bone Marrow Transplant 2016;51:645-53.

2. Beyar-Katz O, Lavi N, RingelsteinHarlev S, et al. Superior outcome of patients with favorable-risk acute myeloid leukemia using consolidation with autologous stem cell transplantation. Leuk Lymphoma 2019; 60:2449-56.

3. Lazarus HM, El Jurdi N. Autologous hematopoietic cell transplantation for adult acute myeloid leukemia: An obsolete or resurfacing concept? Best Pract Res Clin Haematol 2017;30:32732.

4. Heini AD, Berger MD, Seipel K, et al. Consolidation with autologous stem cell transplantation in first remission is safe and effective in AML patients above 65 years. Leuk Res 2017;53:28-34.

5. Czerw T, Labopin M, Gorin NC, et al. Long-term follow-up of patients with acute myeloid leukemia surviving and free of disease recurrence for at least 2 years after autologous stem cell transplantation: A report from the Acute Leukemia Working Party of the European Society for Blood and Marrow Transplantation. Cancer 2016;122:18807.

6. Saraceni F, Bruno B, Lemoli RM, et al. Autologous stem cell transplantation is still a valid option in good- and intermediate-risk AML: a GITMO survey on 809 patients autografted in first complete remission. Bone Marrow Transplant 2017;52:163-6.

7. Miyamoto T, Nagafuji K, Fujisaki T, et al. Prospective randomization of postremission therapy comparing autologous peripheral blood stem cell transplantation versus high-dose cytarabine consolidation for acute myelogenous leukemia in first remission. Int J Hematol 2018;107:46877.

8. Li Z, Liu Y, Wang Q, et al. Autologous stem cell transplantation is a viable postremission therapy for intermediaterisk acute myeloid leukemia in first complete remission in the absence of a matched identical sibling: A metaanalysis. Acta Haematol 2019;141:164-75.

9. Döhner H, Estey E, Grimwade D, et al. Diagnosis and management of AML in adults: 2017 ELN recommendations from an international expert panel. Blood 2017;129:424-7.

10. Vellenga E, Van Putten W, Ossenkoppele GJ, et al. Autologous peripheral blood stem cell transplantation for acute myeloid leukemia. Blood 2011;118:6037-42.

11. Cioch M, Jawniak D, Wach M, et al. Autologous hematopoietic stem cell transplantation for adults with acute myeloid leukemia. Transplant Proc 2016;48:1814-7.

12. Christopeit M, Labopin M, Gorin NC, et al. Allogeneic stem cell transplantation following relapse post autologous stem cell transplantation in adult patients with acute myeloid leukemia: A retrospective analysis of 537 patients from the Acute Leukemia Working Party of the EBMT. Am J Hematol 2018;93:1532-42.

13. Yoon JH, Kim HJ, Park SS, et al. Clinical outcome of autologous hematopoietic cell transplantation in adult patients with acute myeloid leukemia: Who may benefit from autologous hematopoietic cell transplantation? Biol Blood Marrow Transplant 2017;23:588-97.

14. Limvorapitak W, Barnett MJ, Hogge $\mathrm{DE}$, et al. Outcomes of intermediate risk karyotype acute myeloid leukemia in first remission undergoing autologous stem cell transplantation compared with allogeneic stem cell transplantation and chemotherapy consolidation: A retrospective, propensity-score adjusted analysis. Clin Lymphoma Myeloma Leuk 2018;18:e481-e491.

15. Mizutani M, Takami A, Hara M, et al. Comparison of autologous and unrelated transplants for cytogenetically normal acute myelogenous leukemia. Biol Blood Marrow Transplant 2017;23:1447-54.

16. Baron F, Efficace F, Cannella L, et al. Long-term follow-up of a trial comparing post-remission treatment 
with autologous or allogeneic bone marrow transplantation or intensive chemotherapy in younger acute myeloid leukemia patients. Haematologica 2019;105:13-6.

17. Saraceni F, Labopin M, Gorin NC, et al. Matched and mismatched unrelated donor compared to autologous stem cell transplantation for acute myeloid leukemia in first complete remission: a retrospective, propensity score-weighted analysis from the ALWP of the EBMT. J Hematol Oncol 2016;9:79.

18. Mannis GN, Martin TG, Damon LE, et al. Long-term outcomes of patients with intermediate-risk acute myeloid leukemia treated with autologous hematopoietic cell transplant in first complete remission. Leuk Lymphoma 2016;57:1560-6. 